\title{
24,25-Dihydroxyvitamin D Measurement
}

National Cancer Institute

\section{Source}

National Cancer Institute. 24,25-Dihydroxyvitamin D Measurement. NCI Thesaurus.

Code C156511.

The determination of the amount of 24,25-dihydroxyvitamin D present in a sample. 